\title{
MICROMECHANICAL MODELLING OF DAMAGE INDUCED BY DELAYED ETTRINGITE FORMATION
}

\author{
ABDO YAMMINE*, FRANCOIS BIGNONNET, \\ NORDINE LEKLOU, MARTA CHOINSKA \\ AND TERESA STRYSZEWSKA** \\ *Institut de Recherche en Génie Civil et Mécanique GeM - UMR CNRS 6183, Université Bretagne \\ Loire, Université de Nantes, IUT Saint Nazaire, 58 rue Michel Ange, 44600 Saint-Nazaire, France \\ e-mail: abdo.yammine@univ-nantes.fr \\ ${ }^{* *}$ Cracow University of Technology, Faculty of Civil Engineering \\ Ul. Warszawska 24, 31-155 Kraków, Poland
}

Key words: internal sulfate attack, damage, multi-scale modelling, durability, poromechanics

\begin{abstract}
Delayed ettringite formation (DEF) is a pathology that induces concrete swelling and cracking. Factors contributing to DEF have been identified experimentally, but on the modelling side the link between DEF-induced expansion and the evolution of concrete and mortar physical properties has been less studied. A multi-scale poromechanical model is introduced which features a description of DEF induced mortar damage by progression of microcracking. The model considers mortar as a three phases material that is constituted of cement paste, sand and micro-cracks. The Mori-Tanaka scheme is used to homogenise the poroelastic properties of the mortar. The loading parameters are a macroscopic strain and an expansive free strain induced by DEF in cement paste. Damage progression by micro cracking is accounted for by a Griffith-like energy criterion. The model allows to link the DEF-induced expansion at both the microscopic and macroscopic scales to the loss of stiffness of the mortar. A comparison of the model to experimental data on mortars formulated with CEM I cement and natural silica sand is carried out.
\end{abstract}

\section{INTRODUCTION}

\subsection{The DEF problem}

Concrete damage by internal sulfate attack may mostly occur in concrete structures that have been subjected to early elevated temperature above $65 \sim 70^{\circ} \mathrm{C}$ and conditioned in high moisture environments or water [1]. Water and moisture are required for the development of $\mathrm{DEF}$, as ettringite formation needs water that is absorbed from the surrounding environment [2].

Many experimental studies have established a link between ettringite formation and the loss of concrete mechanical properties. Com- pressive strength and Young modulus decrease when DEF-induced expansion exceeds $0.04 \%$ in concrete and mortar [3]. Moreover, a few studies have highlighted transfer properties evolution of concrete and mortar impacted by DEF development. Delayed ettringite occurs when early ettringite crystallisation is inhibited by physical and chemical conditions of concrete mixture. Damidot and Glasser have elaborated $\mathrm{CaO} \cdot \mathrm{Al}_{2} \mathrm{O}_{3} \cdot \mathrm{CaSO}_{4} \cdot \mathrm{H}_{2} \mathrm{O}$ system stability in aqueous solution at different temperatures and $\mathrm{pH}$ and found that ettringite is less stable at high temperature $\left(50\right.$ to $\left.85^{\circ} \mathrm{C}\right)$ than at normal ambient temperature $\left(25^{\circ} \mathrm{C}\right)[4,5]$. On the oppo- 
site side, calcium monosulfoaluminate (Afm) is more stable than ettringite (Aft) at high temperatures. When the mixture is exposed to elevated temperatures, almost all ettringite that should be formed is converted into monosulfate and excess sulfate ions are liberated into the mixture according to equation (1).

$$
\begin{gathered}
\underbrace{3 \mathrm{CaO} \cdot \mathrm{Al}_{2} \mathrm{O}_{3} \cdot 3 \mathrm{CaSO}_{4} \cdot 32 \mathrm{H}_{2} \mathrm{O}}_{\text {ettringite }} \frac{\mathrm{Al}_{2} \mathrm{O}_{3} \cdot \mathrm{CaSO}_{4} \cdot 12 \mathrm{H}_{2} \mathrm{O}}{\underset{\text { monosulfoaluminate }}{\stackrel{\text { when } \uparrow}{\mathrm{CaO}} \downarrow}} \\
\quad+2 \mathrm{Ca}^{2+}+2 \mathrm{SO}_{4}{ }^{2-}+20 \mathrm{H}_{2} \mathrm{O}
\end{gathered}
$$

\subsection{Process and symptomps of DEF}

DEF is an expansive process, marked by expansion of concrete and eventually the development of large cracks [6]. The process of damage progression is till today not very wellestablished. Four theoretical explanations have been proposed as the root cause for expansion and crack progression. These theories are :

a) Expansion of colloidal ettringite by water absorption [7].

b) The phenomenon of double electric layer [8].

c) Uniform isotropic expansion of cement paste due to crystallization of ettringite in $\mathrm{C}-\mathrm{S}-\mathrm{H}$ micropores [9] .

d) Crystallization of large ettringite crystals in macropores, cracks and paste/aggregates interfaces [6].

The first two theories have been almost refuted and experimental studies tend to confirm the $3^{\text {rd }}$ or the $4^{\text {th }}$ theory [10]. According to experimental studies and to MEB images, the last two theories are both probable and may be combined in one theory as Brunetaud proposed [11]. As the crystallisation pressure is higher in small pores, the small capillary pores are filled with ettringite and elevated crystallisation pressures develop on a microscopic level in the cement paste [12]. Aggregates prevent free expansion of the paste, developing microscopic tensile stresses in some parts of the paste and inducing cracking or pre-DEF cracks enlargement. In a second step ettringite crystal ions diffuse from small pores to larger ones where crystallization pressure is weaker. As the paste had been weakened by cracks development, small crystallization pressure is able to continue the expansion. Brunetaud proposed that macroscopic expansion is induced by crystallisation pressures in small pores till $0,1 \% \sim 0,4 \%$ of macroscopic expansion. The second phase will start at $0,4 \%$ of macroscopic expansion. The two phases likely coexist during a transition period.

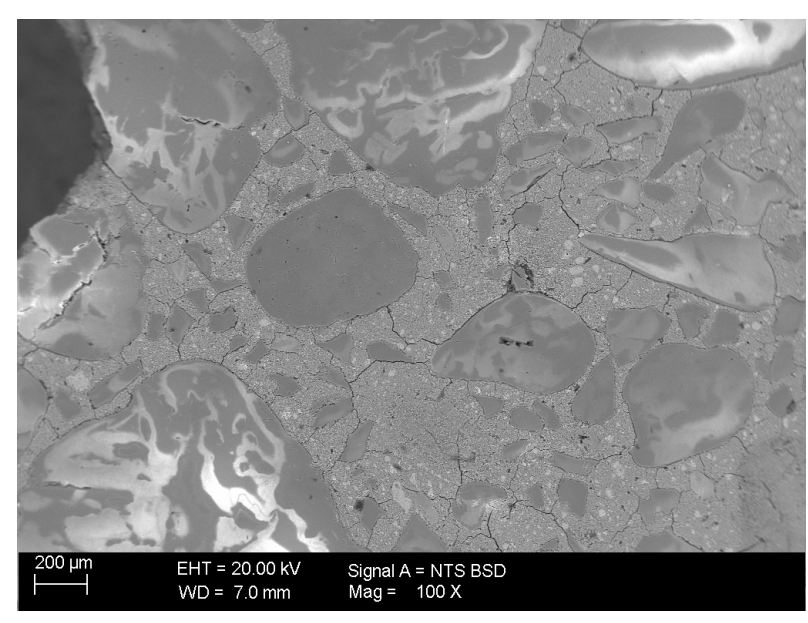

Figure 1: SEM image for 180 days of immersion in water for Normalized CEM I (Y) ${ }^{\text {tab } 1}$ mortar at $0.3 \%$ DEF induced expansion.

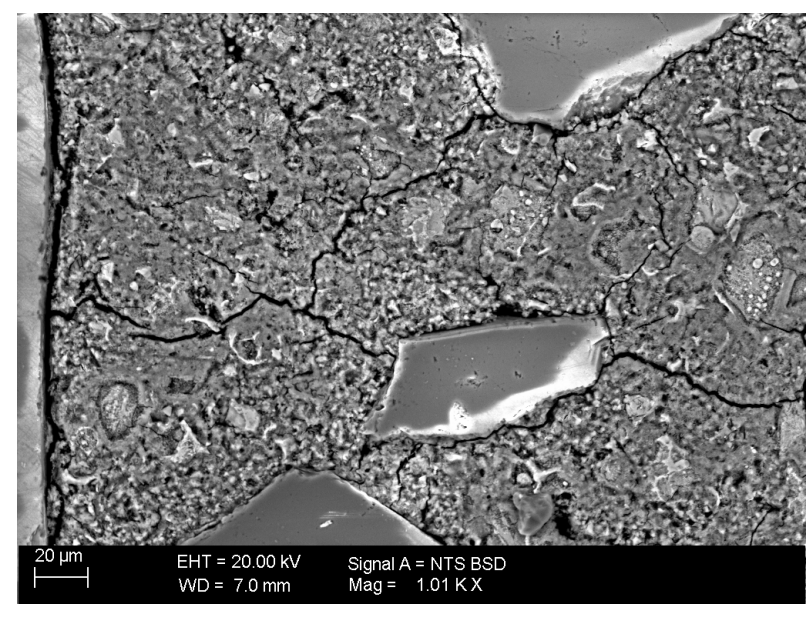

Figure 2: SEM image for the same specimen in Fig 1 at a higher magnification of $1 \mathrm{~K}$ times. 
The SEM image in Fig.1 magnified at 100 times is for a normalized mortar which is subject to DEF damage. The sample has been immersed for 180 days in water and had a macroscopic expansion of $0.3 \%$. A micro-crack network develops in the cement paste and at some interfaces. The length of cracks is around a few hundred micrometers. For the same specimen, magnification at 1000 times in Fig.2 shows clearly at a finer level crack openings estimated to be around $1 \sim 3 \mu \mathrm{m}$.

\section{POROMECHANICAL MODEL WITH DAMAGE PROGRESSION}

Notations: We will use in our calculus the isotropic $4^{\text {th }}$ order tensors $\mathbb{I}, \mathbb{I}$ and $\mathbb{K}$ where $\mathbb{I}$ is the identity, $\mathbb{J}$ is the spherical projector and $\mathbb{K}$ the deviatoric projector that verify the properties $\mathbb{I}=\mathbb{J}+\mathbb{K}, \mathbb{J}=\frac{1}{3} \mathbf{1} \otimes \mathbf{1}$ and $\mathbb{K}=\mathbb{I}-\mathbb{J}$, with 1 is the identity $2^{\text {nd }}$ order tensor. The tensors $\mathbb{J}$ and $\mathbb{K}$ are orthogonal and idempotent for the : product, and any isotropic $4^{\text {th }}$ order tensor $\mathbb{T}$ admits a unique decomposition $\mathbb{T}=T_{1} \mathbb{J}+T_{2} \mathbb{K}$.

\subsection{Model characteristics}

Homogenization is a procedure to obtain at the macroscopic scale the effective mechanical properties of a material which is heterogeneous at the microscopic scale. The process requires information on the different constituents of the heterogeneous material such as their mechanical constitutive law and their morphological organization.

Our model focuses on mortar, which is composed of three phases at the microscopic scale: hydrated cement paste, sand particles and micro-cracks (see figure 3). The model considers that DEF swelling is the consequence of an isotropic expansion in the cement paste according to the theory in $1.2 \mathrm{c}$ ). The progression of DEF development will be driven by the free expansion of cement paste $\left(\varepsilon_{f}\right)$. Time is not explicitly accounted for in the model as chemical and diffusion phenomena are not detailed. Chemo-diffusive phenomenon, which occur at a finer scale, will be studied in a future model.

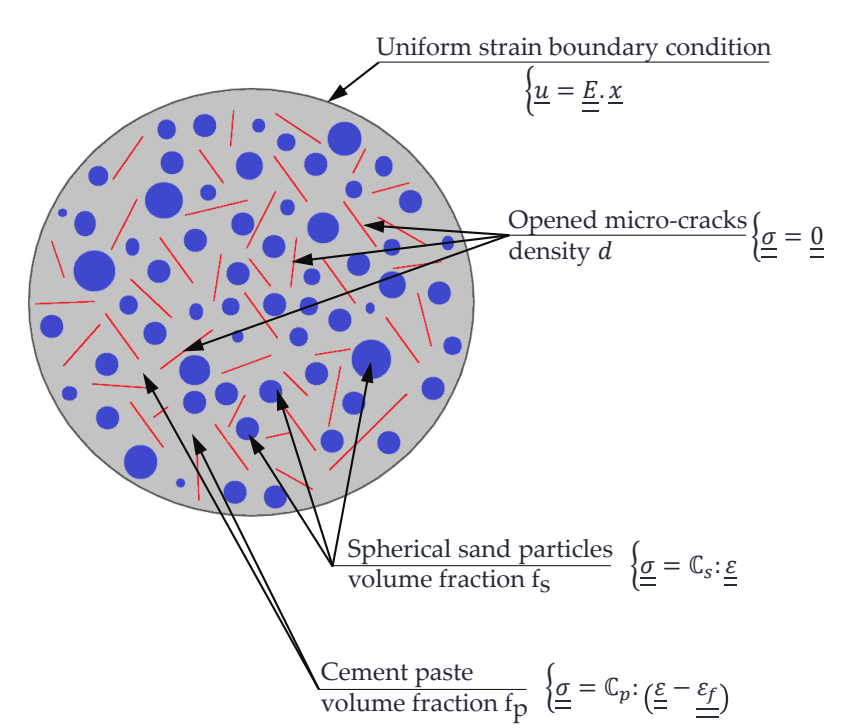

Figure 3: Mortar model with the three phases : cement paste, sand particles and cracks.

The local constitutive law of sand grains and cement paste is assumed isotropic linear elastic with compressive moduli $k_{s} ; k_{p}$ and shear moduli $\mu_{s} ; \mu_{p}$ respectively. The corresponding stiffness tensors $\left(\mathbb{C}_{s}, \mathbb{C}_{p}\right)$ are:

$$
\begin{aligned}
& \mathbb{C}_{s}=3 k_{s} \mathbb{J}+2 \mu_{s} \mathbb{K} \\
& \mathbb{C}_{p}=3 k_{p} \mathbb{J}+2 \mu_{p} \mathbb{K}
\end{aligned}
$$

The DEF-driven expansive free strain in cement paste is an isotropic swelling $\underline{\underline{\varepsilon}}_{f}=\varepsilon_{f} \underline{\underline{1}}$. Sand grains are modelled with a spherical shape within the cement paste matrix. The volume fraction of the sand grains and the cement paste are denoted $f_{s}$ and $f_{p}$ respectively.

Cracks are represented as penny-shaped spheroids with an isotropic distribution of orientations. To model cracks, the aspect ratio $\omega=b / a$ of the oblate spheroids is considered in the limiting case $\omega \rightarrow 0$, where $b$ and $a$ are the thickness and radius of the spheroids. The volume fraction of cracks $f_{c}=\frac{4 \pi}{3} a^{2} b N$ is vanishing, where $N$ is the number of cracks per unit volume. The extent of micro-cracking is hence represented by the crack density parameter $d=N a^{3}$ introduced by Budiansky and O'Connell [13].

In a representative volume element (RVE) of mortar, $\underline{z}$ denotes the position vector which 
could be located in the sand, the cement paste or a crack. The displacement field $\underline{u}$ verifies uniform strain boundary conditions $\underline{u}=\underline{\underline{E}} \cdot \underline{z}$, where $\underline{\underline{E}}$ is the macroscopic strain tensor. The microscopic strain field $\underline{\underline{\varepsilon}}$ is then related to the macroscopic strain by the strain averaging rule $\underline{\underline{E}}=\underline{\bar{\varepsilon}}$, where $\bar{\bullet}$ denotes the volume average over the RVE of a field $\bullet$. The local stress $\underline{\sigma}$ is obtained from the strain and the free strain using the local constitutive laws (eq. (4)). To sum up, the homogenization problem $(\mathbf{P})$ is defined by the following boundary value problem:

$$
\mathbf{P} \begin{cases}\underline{\text { div }} \underline{\underline{\sigma}}=\underline{0} & \text { at any point } \\ \underline{\underline{\varepsilon}}=\underline{\underline{\underline{\underline{\nu}}}}=\underline{\underline{\underline{E}}} \cdot \underline{\underline{z}} & \text { at any point } \\ \underline{\underline{\sigma}}=\underline{\underline{0}} & \text { on the edge } \\ \underline{\underline{\sigma}}=\mathbb{C}_{s}: \underline{\underline{\varepsilon}} & \text { in cracks } \\ \underline{\underline{\sigma}}=\mathbb{C}_{p}:\left(\underline{\underline{\varepsilon}}-\underline{\underline{\varepsilon}}_{f}\right) & \text { in pand }\end{cases}
$$

The two loading parameters of problem $(\mathbf{P})$ are the macroscopic strain $\underline{\underline{E}}$ and the expansive free strain $\varepsilon_{f}$ in cement paste. By superposition, the problem $(\mathbf{P})$ is divided into two problems $\left(\mathbf{P}^{\prime}\right)$ and (P"):

- (P') : no free expansion in the cement paste $\varepsilon_{f}^{\prime}=0$ and imposed macroscopic strain $\underline{\underline{E}}^{\prime}=\underline{\underline{E}}$

- (P") : no macroscopic strain $\underline{\underline{E}}^{\prime \prime}=0$ and imposed free expansion in the cement paste $\varepsilon_{f}^{\prime \prime}=\varepsilon_{f}$.

In order to ease the analogy with poro-elasticity in what follows, let us denote:

$$
p=k_{p} \varepsilon_{f}
$$

the pressure that would arise in a confined element of cement paste subjected to an expansive free strain $\varepsilon_{f}$ but zero total strain.

$$
\mathbf{P}^{\prime} \begin{cases}\underline{\operatorname{div}} \underline{\underline{\sigma^{\prime}}=\underline{0}} & \text { at any point } \\ \underline{\underline{\varepsilon^{\prime}}}=\underline{\underline{\nabla^{s}}} \underline{\underline{u^{\prime}}} \cdot \underline{\underline{\underline{E}}} \cdot \underline{\text {z}} & \text { at any point } \\ \underline{\underline{\sigma}}^{\prime}=\underline{\underline{0}} & \text { in cracks edge } \\ \underline{\underline{\sigma^{\prime}}}=\mathbb{C}_{s}: \underline{\underline{\varepsilon^{\prime}}} & \text { in sand } \\ {\underline{\underline{\sigma^{\prime}}}}^{\prime}=\mathbb{C}_{p}: \underline{\underline{\varepsilon^{\prime}}} & \text { in paste }\end{cases}
$$

$$
\mathbf{P}, \begin{cases}\underline{\operatorname{div}} \underline{\underline{\sigma^{\prime \prime}}=\underline{0}} & \text { at any point } \\ \underline{\underline{\varepsilon}}^{\prime \prime}=\underline{\underline{\nabla}}^{s} \underline{u}^{\prime \prime} & \text { at any point } \\ \underline{u}^{\prime \prime}=\underline{\underline{0}} & \text { on the edge } \\ \underline{\underline{\sigma}}^{\prime \prime}=\underline{\underline{0}} & \text { in cracks } \\ \underline{\underline{\sigma}}^{\prime \prime}=\mathbb{C}_{s}: \underline{\underline{\varepsilon}}^{\prime \prime} & \text { in sand } \\ \underline{\underline{\sigma}}^{\prime \prime}=\mathbb{C}_{p}: \underline{\underline{\varepsilon}}^{\prime \prime}-p \underline{\underline{1}} & \text { in paste }\end{cases}
$$

\subsection{Concentration and homogenization}

By linearity of the problems at hands, the local strain fields $\underline{\underline{\varepsilon}}^{\prime}$ and $\underline{\underline{\varepsilon}}^{\prime \prime}$ in the two problems (P') and (P') can be formally obtained from the two loading parameters $\underline{E}$ and $p$ using two localisation tensor fields $\mathbb{A}(z)$ and $\underline{\underline{a}}(z)$ :

$$
\begin{aligned}
& \underline{\underline{\varepsilon}}^{\prime}=\mathbb{A}(z): \underline{\underline{E}} \\
& \underline{\underline{\varepsilon}}^{\prime \prime}=-\underline{\underline{a}}(z) p
\end{aligned}
$$

Next, the homogenization step involves volume averages of the strain and stress fields over the RVE. First, the stress averaging rule re-

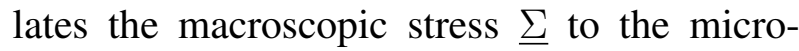
scopic stress field $\underline{\underline{\sigma}}=\underline{\underline{\sigma}}^{\prime}+\underline{\underline{\sigma}}^{\prime \prime}$ by $\underline{\underline{\underline{\Sigma}}}=\overline{\underline{\sigma}}$. Second, since the model involves a free strain in the cement paste, the Lagrangian variation $\Delta f_{p}$ of cement paste volume fraction is the second macroscopic variable that needs to be determined. This quantity can be understood as to the variation of the Lagrangian porosity in poroelasticity. To sum up, the two macroscopic variable sought for are:

$$
\left\{\begin{aligned}
\underline{\underline{\Sigma}} & =\overline{\underline{\sigma}}=\underbrace{\overline{\sigma^{\prime}}}_{\mathbf{P}}+\underbrace{\overline{\sigma^{\prime \prime}}}_{\mathbf{P} ”} \\
\Delta f_{p} & =\overline{I_{p} \underline{\underline{1}}: \underline{\underline{\varepsilon}}^{\underline{\sigma^{\prime}}}} \\
& =\overline{I_{p} \underline{\underline{1}}: \underbrace{\underline{\varepsilon^{\prime}}}_{\mathbf{P}}}+\overline{I_{p} \underline{\underline{1}}: \underbrace{\underline{\underline{\varepsilon}}^{\prime \prime}}_{\mathbf{P}}}
\end{aligned}\right.
$$

where $I_{p}$ is the indicator function of cement paste defined as :

$$
I_{p}(z)=\left\{\begin{array}{l}
1 \text { if } z \in \text { paste } \\
0 \text { else }
\end{array}\right.
$$

so that its volume average over the RVE is equal to the paste volume fraction $\overline{I_{p}}=f_{p}$. 
Gathering equations (6, 7, 8, 9 and 10, we obtain:

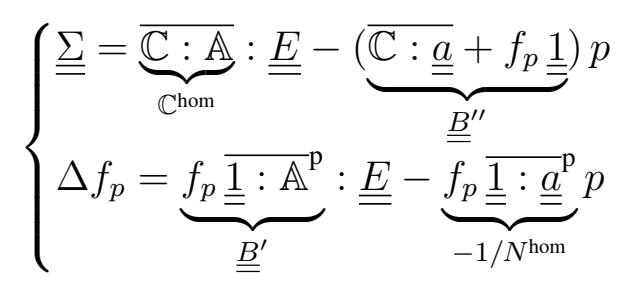

where $\bar{\bullet}^{p}=\overline{I_{p} \bullet} / f_{p}$ denotes the volume average over the cement paste phase of a field $\bullet$. Following [14], application Hill's lemma can be used to prove that $\underline{\underline{B}}^{\prime \prime}=\underline{\underline{B}}^{\prime}\left(=\underline{\underline{B}}^{\text {hom }}\right)$. In (12), $\mathbb{C}^{\text {hom }}$ appears as the homogenized stiffness tensor, while $\underline{\underline{B}}^{\text {hom }}$ and $N^{\text {hom }}$ are homogenized poro-elastic properties which are the analogue to the Biot tensor and the Biot modulus respectively in poro-elasticity.

Finally, the homogenized constitutive law is obtained from eq.(12):

$$
\begin{cases}\underline{\underline{\underline{E}}} & =\mathbb{C}^{\text {hom }}: \underline{\underline{E}}-\underline{\underline{B}}^{\text {hom }} p \\ \Delta f_{p} & =\underline{\underline{B}}^{\text {hom }}: \underline{\underline{E}}+\frac{1}{N^{\text {hom }}} p\end{cases}
$$

\subsection{Mori-Tanaka estimate of the homoge- nized poro-elastic parameters}

The determination of the homogenized poromechanical properties $\mathbb{C}^{\text {hom }}, \underline{B}^{\text {hom }}$ and $N^{\text {hom }}$ requires knowledge of appropriate averages of the concentration tensors $\mathbb{A}(z)$ and $\underline{\underline{a}}(z)$. The matrix-inclusion morphology of mortar suggests to resort to the Mori-Tanaka scheme to estimate these concentration tensors. The application of Mori-Tanaka scheme to problem $\left(\mathbf{P}^{\prime}\right)$ is rather classical but will be first recalled in order to ease its generalization to problem (P'").

\subsubsection{Problem (P')}

In the Mori-Tanaka scheme, the average deformation in the cement paste matrix ${\underline{\bar{\varepsilon}^{\prime}}}^{p}$ is used an auxiliary deformation $\underline{\underline{E}}_{0}^{\prime}$, i.e. ${\underline{\underline{E^{\prime}}}}_{0}^{\prime=}{\overline{\underline{\varepsilon}^{\prime}}}^{p}$. The average strain ${\overline{\varepsilon^{\prime}}}^{i}$ in each inclusion $i$ (sand or crack) is then estimated via the Eshelby problem with a far field deformation $\underline{\underline{E}}_{0}^{\prime}$ as:

$$
\begin{aligned}
{\frac{{\underline{\varepsilon^{\prime}}}^{i}}{=}}^{i}=\mathbb{L}^{i}:{\underline{\underline{E^{\prime}}}}_{0}^{\prime} \\
\text { with } \mathbb{L}^{i}=\left[\mathbb{I}+\mathbb{P}_{p}^{i}:\left(\mathbb{C}_{i}-\mathbb{C}_{p}\right)\right]^{-1}
\end{aligned}
$$

where $\mathbb{P}_{p}^{i}$ is the Hill tensor of an inclusion $i$ within the cement paste matrix. For sand grains modeled as spheres, the Hill tensor is

$$
\begin{aligned}
\mathbb{P}_{p}^{s} & =\frac{\alpha}{3 k_{p}} \mathbb{J}+\frac{\beta}{2 \mu_{p}} \mathbb{K} \quad \text { with } \\
\alpha & =\frac{3 k_{p}}{3 k_{p}+4 \mu_{p}} \quad ; \quad \beta=\frac{6}{5} \frac{k_{p}+2 \mu_{p}}{3 k_{p}+4 \mu_{p}}
\end{aligned}
$$

so that the components in the $(\mathbb{J}, \mathbb{K})$ basis of the concentration tensor of a sand grain in the Eshelby problem are

$$
\begin{aligned}
& L_{1}^{s}=\left[1+\alpha\left(k_{s} / k_{p}-1\right)\right]^{-1} \\
& L_{2}^{s}=\left[1+\beta\left(\mu_{s} / \mu_{p}-1\right)\right]^{-1}
\end{aligned}
$$

In the limiting case of the infinitely flat cracks $(\omega \rightarrow 0)$, the tensor $\mathbb{L}^{c}$ diverges as $1 / \omega$ while the volume fraction of crack $f_{c}$ vanishes as $\omega$, so that the total contribution of the isotropically distributed opened cracks to the average strain is finite and given as a function of the crack density parameter by [15]:

$$
\lim _{\omega \rightarrow 0} f_{c}{\overline{\mathbb{L}^{c}}}^{c}=d \mathbb{Q}
$$

where the components of $\mathbb{Q}$ in the $(\mathbb{J}, \mathbb{K})$ basis are:

$$
\begin{aligned}
Q_{1} & =\frac{4 k_{p}\left(3 k_{p}+4 \mu_{p}\right)}{3 \mu_{p}\left(3 k_{p}+\mu_{p}\right)} \\
Q_{2} & =Q_{1} \frac{4 \mu_{p}\left(9 k_{p}+4 \mu_{p}\right)}{15 k_{p}\left(3 k_{p}+2 \mu_{p}\right)}
\end{aligned}
$$

The auxiliary deformation $\underline{\underline{E}}_{0}^{\prime}$ is determined using the strain averaging rule $\frac{\underline{\underline{\varepsilon^{\prime}}}}{\underline{\underline{E^{\prime}}}}=\underline{\underline{E^{\prime}}}$, which yields:

$$
\underline{\underline{E}}^{\prime}=\left(f_{p} \mathbb{I}+f_{s} \mathbb{L}^{s}+d \mathbb{Q}\right): \underline{\underline{E}}_{0}^{\prime}
$$

Since $\underline{\underline{E}}_{0}^{\prime}={\overline{\varepsilon^{\prime}}}^{p}$, the average of the strain concentration tensor in the cement paste is:

$$
\overline{\mathbb{A}}^{p}=\left(f_{p} \mathbb{I}+f_{s} \mathbb{L}^{s}+d \mathbb{Q}\right)^{-1}
$$

Gathering eqs. (14), (19) and (20) yields the average of the strain concentration tensor in the sand:

$$
\overline{\mathbb{A}}^{s}=\mathbb{L}^{s}: \overline{\mathbb{A}}^{p}
$$


The homogenized stiffness tensor is defined in the first line of $(12)$ as $\mathbb{C}^{\text {hom }}=\overline{\mathbb{C}: \mathbb{A}}$. Its Mori-Tanaka estimate is isotropic and expressed in the form $\mathbb{C}^{\text {hom }}=3 k^{\text {hom }} \mathbb{J}+2 \mu^{\text {hom }} \mathbb{K}$ where $k^{\text {hom }}$ and $\mu^{\text {hom }}$ are the homogenized bulk and shear moduli. Gathering eqs. (16) and (20) gives:

$$
\begin{aligned}
& k^{\mathrm{hom}}=\left(f_{p} k_{p}+f_{s} k_{s} L_{1}^{s}\right) \bar{A}_{1}^{p} \\
& \mu^{\mathrm{hom}}=\left(f_{p} \mu_{p}+f_{s} \mu_{s} L_{2}^{s}\right) \bar{A}_{2}^{p}
\end{aligned}
$$

Similarly, the analogue to the Biot tensor can be obtained from the second line of (12) as $\underline{\underline{B}}^{\text {hom }}=f_{p} \overline{\underline{1}}: \mathbb{A}^{\mathrm{p}}$. Its Mori-Tanaka estimate is isotropic and given by $\underline{\underline{B}}^{\text {hom }}=b^{\text {hom }} \underline{\underline{1}}$ with:

$$
b^{\text {hom }}=f_{p} \bar{A}_{1}^{p}
$$

\subsubsection{Problem (P')}

We still have to determine the strain concentration tensor $\underline{a}$ of problem (P') in order to obtain $N^{\text {hom }}$ defined in the second line of (12). By superposition, the Mori-Tanaka estimate of this problem with a pre-stress in the matrix involves Eshelby problems with an opposite pre-stress in the inclusions. Denoting ${\underline{\underline{E^{\prime \prime}}}}_{0}^{\prime \prime}={\underline{\underline{\varepsilon}^{\prime \prime}}}^{p}$ the auxiliary strain, which is set equal to the average strain in the cement paste, the Mori-Tanaka estimate of the concentration tensors in an inclusion $i$ (sand or crack) is:

$$
{\overline{\underline{\varepsilon^{\prime \prime}}}}^{i}=\mathbb{L}^{i}:\left(\underline{\underline{E}}_{0}^{\prime \prime}-\mathbb{P}_{p}^{i}: p \underline{\underline{1}}\right)
$$

Since the macroscopic strain $\underline{\underline{E}}^{\prime \prime}=0$ in problem $\left(\mathbf{P}\right.$ '), the strain averaging rule $\overline{\underline{\varepsilon^{\prime \prime}}}=0$ allows to retrieve the auxiliary strain as:

$$
\begin{aligned}
\underline{\underline{E}}_{0}^{\prime \prime} & =\overline{\mathbb{A}}^{p}: \mathbb{Y}: \underline{\underline{1}} p \quad \text { where } \\
\mathbb{Y} & =f_{s} \mathbb{L}^{s}: \mathbb{P}_{p}^{s}+d \mathbb{Q}: \mathbb{C}_{p}^{-1}
\end{aligned}
$$

Using both eqs.(25) and (9), one obtains the average strain concentration tensor in cement paste $\underline{\underline{\underline{a}}}^{p}$ :

$$
\underline{\underline{\bar{a}}}^{p}=-\overline{\mathbb{A}}^{p}: \mathbb{Y}: \underline{\underline{1}}
$$

The analogue to the Biot modulus $N^{\text {hom }}$ can then be estimated from eqs. (12) and (26) as:

$$
\frac{1}{N^{\mathrm{hom}}}=-f_{p} \underline{\underline{1}}: \underline{\underline{\underline{a}}}^{p}=3 f_{p} \bar{A}_{1}^{p} Y_{1}=3 b^{\mathrm{hom}} Y_{1}
$$

Note that the first line of (9) gives another way to determine $\underline{\underline{B}}^{\text {hom }}$ from the strain concentration tensor of problem (P'). However, as shown in [16], in this case Mori-Tanaka scheme is known to break the symmetry requirement $\underline{B}^{\prime}=\underline{B}^{\prime \prime}$. This shortcoming is not investigated further herein, and expression (23) will be used to estimate $\underline{B}^{\text {hom }}$ in what follows.

\subsection{Damage description and propagation}

The homogenization procedure detailed in sec. 2.3 gives explicit expressions of the homogenized stiffness tensor $\mathbb{C}^{\text {hom }}(22)$ and the homogenized poro-elastic parameters $\underline{\underline{B}}^{\text {hom }}$ and $N^{\text {hom }} 27$ as a function of the crack density parameter $d$. In what follows, the crack density parameter $d$ will be considered as the unique variable which describes damage state [17]. The damage evolution law will be described by a Griffith-like energy criterion.

Following Dormieux and Kondo [17], the macroscopic density of stored elastic energy $\Psi$ in the mortar is a quadratic function of the two loading parameters $\underline{\underline{E}}$ and $p=k_{p} \epsilon_{f}$ :

$$
\Psi=\frac{1}{2} \underline{\underline{E}}: \mathbb{C}^{\text {hom }}: \underline{\underline{E}}+\frac{p^{2}}{2 N^{\text {hom }}}
$$

The density of potential energy $\Psi^{*}$ is the difference between the density of stored elastic energy and the work of external loads per unit volume of mortar, here due to the free expansive strain $\epsilon_{f}=p / k_{p}$ in the cement paste phase:

$$
\Psi^{*}=\Psi-p \Delta f_{p}
$$

Replacing in eq. (29) the cement paste volume fraction variation $\Delta f_{p}$ by its expression (13) gives the expression of the potential energy as a function of the loading parameters and the ho- 
mogenized poro-elastic properties:

$$
\begin{aligned}
\Psi^{*}(\underline{\underline{E}}, p, d) & =\frac{1}{2} \underline{\underline{E}}: \mathbb{C}^{\text {hom }}(d): \underline{\underline{E}} \\
& -\frac{p^{2}}{2 N^{\operatorname{hom}}(d)}-p \underline{\underline{B}}^{\text {hom }}(d): \underline{\underline{E}}
\end{aligned}
$$

During a reversible evolution, the partial derivatives of $\Psi^{*}(\underline{\underline{E}}, p, d)$ w.r.t. the loading parameters $\underline{E}$ and $-\bar{p}$ allow to retrieve the macroscopic poro-elastic constitutive law (13).

In turn, in the case of an irreversible evolution, the scalar $G=-\partial \Psi^{*} / \partial d$ is considered as the thermodynamic force associated with the damage variable $d$, that is, the driving force of damage. $G$ is the analogue to the energy release rate in linear elastic fracture mechanics (LEFM) [18]. The damage propagation criterion proposed by [17] is the analogue to the Griffith criterion in LEFM:

$$
\begin{aligned}
\mathrm{F}(\underline{\underline{E}}, p, d) & =G-G_{c} \\
& =-\frac{\partial \Psi^{*}}{\partial d}(\underline{\underline{E}}, p, d)-G_{c} \leq 0
\end{aligned}
$$

where $G_{c}$ is an analogue to the Griffith critical energy release rate. However $G_{c}$ may depend from the microstructure and hence from the crack density parameter $d$; several dependencies of $G_{c}$ on $d$ have been considered by previous investigators: constant [17], power law [18] or linear [19]. As such, the determination of $G_{c}$ from experimental measurements is often indirect.

In what follows, we are interested in damage propagation due to DEF in mortar samples subjected to no sizable external stress, i.e. one assumes that the macroscopic stress state is $\underline{\underline{\Sigma}}=0$ throughout the evolution. Since the material remains isotropic, the macroscopic strain is an expansive strain $\underline{\underline{E}}=E \underline{\underline{1}}$ and is given by the macroscopic poro-elastic constitutive law (13) as a function of the free expansion of the cement paste by:

$$
E=\frac{k_{p}}{k^{\mathrm{hom}}(d)} b^{\text {hom }}(d) \epsilon_{f}
$$

In the case of the Mori-Tanaka estimate, the ratio $k^{\text {hom }}(d) / b^{\text {hom }}(d)$ is seen to be independent on $d$ from eqs. (22) and (23), so that the relationship between the macroscopic and the microscopic expansions is linear throughout the evolution.

\section{MODEL COMPARISON TO EXPERI- MENTAL RESULTS}

\subsection{Experimental set up}

Five mortar formulations results subjected to DEF are compared to the model results. Four formulations have been prepared by Nguyen, who studied mineral additions impact on DEF [3, 20]. A last formulation was prepared recently (Normalized CEM I (Y)) for additional results comparison. Cement used for all formulations is a CEM I 52.5 N CE CP2 NF. Preparations are elaborated according the NF EN 196-1 standard. Water to cement ratio is equal to 0.56 for all mortars except the later one for which water to cement ratio is equal to 0.5 . Sodium sulfate is used in order to accelerate and enhance DEF development. The added sulfate dosage is estimated for a total sulfate content of $5 \%$ by cement weight. Table 1 summarizes the mix proportions for the different mortars.

Mortars are cast in $40 \times 40 \times 160 \mathrm{~mm}^{3}$ prism steel moulds. Two brass studs, one on the upper and one the lower square surface of the prisms are used to measure the specimen expansion. After molding, the prisms are placed at $20^{\circ} \mathrm{C}$ for 2 hours and cured in an oven at maximum $80^{\circ} \mathrm{C}$ for over 149 hours in order to simulate heat exposure in precast concrete elements [20]. After heat treatment, specimens are placed in plastic boxes filled with deionized water at $20^{\circ} \mathrm{C}$. Water is frequently changed in order to enhance alkali lixiviation which accelerates ettringite formation. Three specimens from each formulation are measured frequently for expansion and non destructive dynamic Young modulus test using impulse excitation technique.

\subsection{Paste and sand volume fractions}

A normalized sand formulation constituted of $1350 \mathrm{~g}$ of normalised sand, $450 \mathrm{~g}$ of dehydrated cement and $225 \mathrm{~g}$ of water is considered in our model. In order to find the volume 
Table 1: Mortar formulations composition

\begin{tabular}{ccccccc}
\hline \multirow{2}{*}{ Reference } & \multicolumn{5}{c}{ Constituents $(\mathrm{g})$} \\
& Cement & Fly ash & Pouzzolan & Sand & $\mathrm{Na}_{2} \mathrm{SO}_{4}$ & Water \\
\hline Normalized CEM I & 450 & - & - & 1350 & 13.95 & 252 \\
Normalized CEM I (Y) & 450 & - & - & 1350 & 13.26 & 225 \\
Fly Ash(10\%) & 315 & 135 & - & 1350 & 13.95 & 252 \\
Pozzolan (10\%) & 405 & - & 45 & 1350 & 13.95 & 252 \\
Pozzolan (20\%) & 360 & - & 90 & 1350 & 13.95 & 252 \\
\hline
\end{tabular}

fractions of sand and cement paste, their corresponding densities will be fixed respectively : $\rho_{s s}=2.65 \mathrm{~kg} / \mathrm{m}^{3}$ and $\rho_{\text {cement }}=3.16 \mathrm{~kg} / \mathrm{m}^{3}$. Assuming that the total volume of mortar is the sum up of each phase volume apart, volume fractions for normalized sand are $f_{p}=0.42$; $f_{s}=0.58$

\subsection{Paste and sand mechanical properties}

The mechanical properties of the normalized sand are taken equal to the mechanical properties of silica crystals since it is integrally constituted of silica :

$$
k_{s}=35 G P a \quad \mu_{s}=30 G P a
$$

The mechanical properties of the paste are estimated from the study of Helmuth and Turk [21] on CEM I cement paste. The paste is assumed to be fully hydrated with a water to cement ratio equal to 0.5 . The bulk and the shear moduli are:

$$
k_{p_{1}}=16 G P a ; \quad \mu_{p_{1}}=6 G P a
$$

Three of the mortars formulations in Tab, 1 used in the comparison are made up of a mixture of mineral additions with CEM I cement. In order to be able to find the pastes moduli of the fly ash and pozzolan mortars, an inverse method is used. Pre-damage experimental young moduli of mortars are equal to $33 \mathrm{GPa}$ for fly ash, $28 \mathrm{GPa}$ for pozzolan (10\%) and $26.5 \mathrm{GPa}$ for pozzolan (20\%) comparing to $34.5 \mathrm{GPa}$ and 36 GPa for the CEM I mortars. The fly ash mortar moduli are close to CEM I mortars so only two groups of pastes will be used in in the model. The first group represent the CEM I and fly ash mortars and the second group the pozzolan mortars. Volume fractions are the same in the two groups. Assuming the same Poisson's ratio for both pastes, the bulk and the shear moduli of the second group paste are obtained by adjusting the homogenized Young modulus to $27.5 \mathrm{GPa}$. The mortar groups are summarized in table 2 .

\subsection{Results}

The evolution of the homogenized Young modulus with the crack density parameter $d$ is shown in Fig 4. $E^{\text {hom }}$ decreases with $d$ at a higher rate at low $d$ than at high $d$.

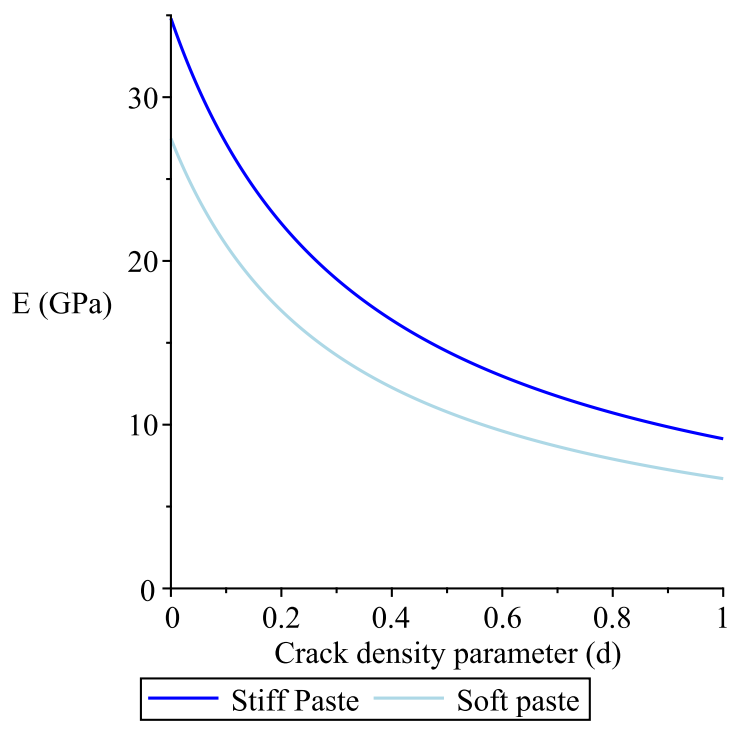

Figure 4: Young modulus in both mortar groups according to crack density evolution

Next, the evolution of damage due to the DEF induced microscopic expansion $\varepsilon_{f}$ is assessed using the damage evolution law (31). Since the macroscopic stress is zero, the macroscopic expansion $E$ is expressed as a function 
Table 2: Mortars groups

\begin{tabular}{lc|cccc}
\hline \multicolumn{1}{c|}{ Experimental } & \multicolumn{5}{c}{ Models } \\
Reference & E (GPa) & Group & E $^{\text {hom }}(\mathrm{GPa})$ & $k_{p}(\mathrm{GPa})$ & $\mu_{p}(\mathrm{GPa})$ \\
\hline Normalized CEM I & 34.5 & & & & \\
Normalied CEM I (Y) & 36 & Stiff paste & 34.8 & 16 & 6 \\
Fly Ash (10\%) & 33 & & & & \\
\hline Pozzolan (10\%) & 28 & Soft paste & 27.5 & \multirow{2}{*}{10.8} & \multirow{2}{*}{4.1} \\
Pozzolan (20\%) & 26.5 & & & & \\
\hline
\end{tabular}

of $\varepsilon_{f}$ by (32). As no direct experimental evaluation of the energy release rate $G_{c}$ is available for the materials under study, several values of $G_{c}$ constant w.r.t. $d$ are considered for simplicity.

The crack density is assumed equal to zero at the beginning of expansion. Then, under the assumption of a constant value of $G_{c}$ throughout the evolution, the onset of damage propagation occurs for a non-zero threshold value of $\varepsilon_{f}$ which depends on the microstructure parameters. Beyond this point, the macroscopic stiffness starts to decrease as expansion increases.

In order to compare the model to observable macroscopic quantities, the loss of macroscopic Young modulus is plotted as a function of the macroscopic expansion in Fig 5, for the experimental results and the model with soft or stiff cement pastes. For each mortar model a set of different $G_{c}$ values are considered. The experimental data trend is best reproduced with $G_{c}$ values in the range 0.25 to $1 \mathrm{MPa}$. The macroscopic expansion value required in the model to trigger damage progression increases as a function of $G_{c}$ and is greater for the soft paste than for the stiff one. In contrast, the experimental data exhibit a loss of Young's modulus right at the onset of the expansion. This suggests that the energy release rate $G_{c}$ may not be constant during the evolution but rather increasing with $d$, as considered for example in [19].

\section{Conclusion}

A micromechanical model of DEF induced damage in mortars has been developed. The assumptions are that damage manifests itself by diffuse micro-cracks at the sand grain scale, but arises as a consequence of a free expansion of the cement paste. The latter is likely driven by pressures due to ettringite crystallization in pores at a finer scale, although this process has not been explicitly described herein. The micro-cracks propagation is described by a Griffith-like energy criterion. These modelling assumptions allow to reproduce macroscopic experimental trends of Young's modulus decrease as a function of the overall expansion. The volume fraction of sand and the stiffness ratio between the sand grains and the cement paste are shown to influence the damage propagation.

Current limitations of the model are that simulated Young's modulus do not trigger right at the onset of expansion, which is due to the assumption of a constant value of the energy release rate. A methodology to assess this energy release rate from independent experimental measures such as three point bending tests remains to be developed, as it is the only unknown parameter of the model.

As a perspective, it would be interesting to extend the present work to model the increase of the transport properties such as permeability and diffusion coefficients with DEF-induced damage.

\section{Aknowlegement}

The authors would like to acknowledge the two projects :

- The Algerian-French cooperation project Tassili 19MDU216;

- The financial support of the Polish National 


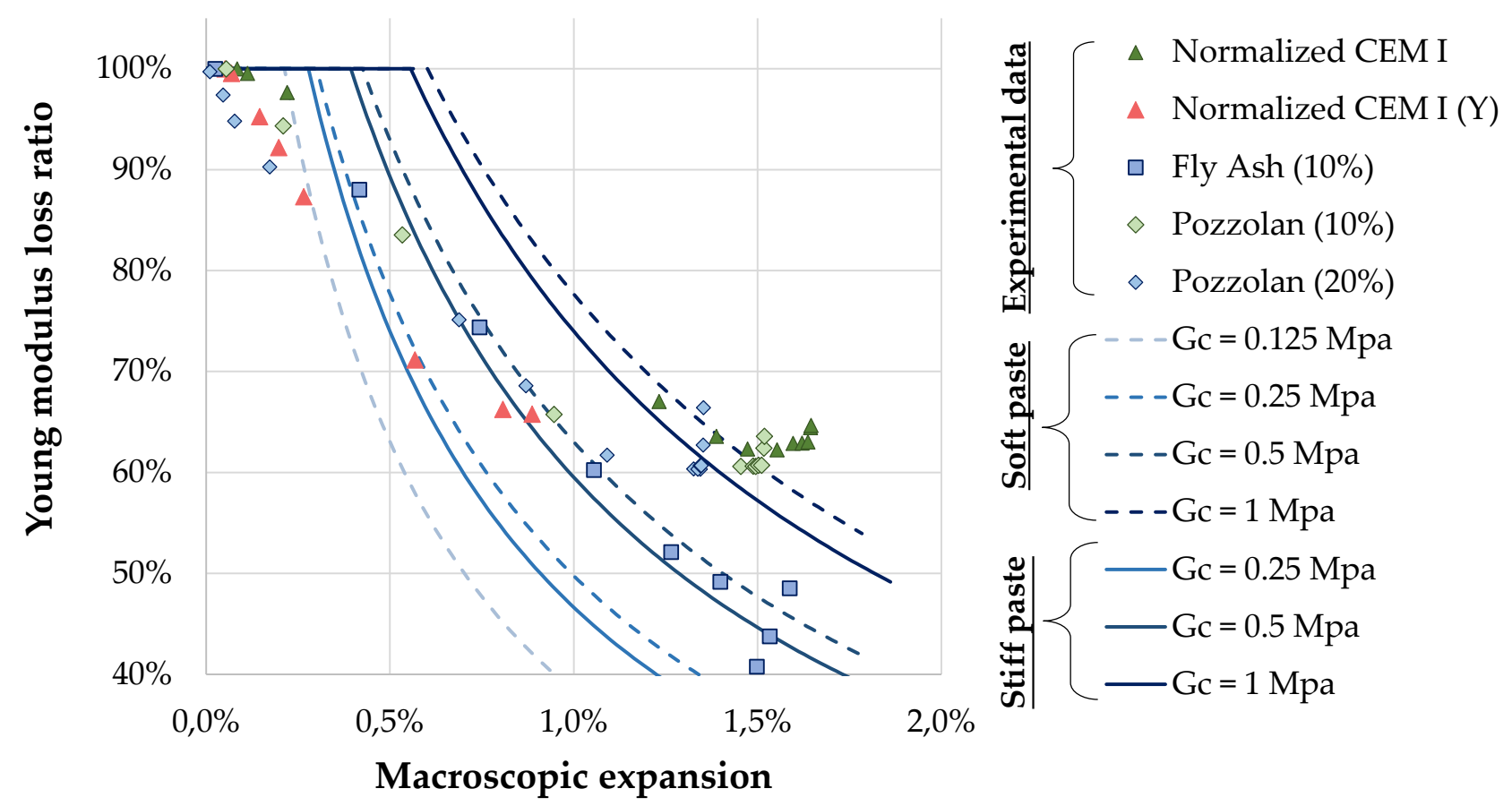

Figure 5: Comparision of experimental results with the model.

Agency for Academic Exchange under the International Academic Partnership Programme: PPI/APM/2018/1/00027 E-mobility and sustainable materials and technologies EMMAT.

\section{REFERENCES}

[1] K. L. Scrivener, D. Damidot, and C. Famy. Possible Mechanisms of Expansion of Concrete Exposed to Elevated Temperatures During Curing (Also Known as DEF) and Implications for Avoidance of Field Problems. Cement, Concrete and Aggregates, 21(1):93, 1999.

[2] C. Famy, K. L. Scrivener, and A. R. Brough. Role of Microstructural Characterisation in Understanding the Mechanism of Expansion due to DEF. In K L Scrivener and J Skalny, editors, International RILEM TC 186-ISA, pages 7-9. 2002.

[3] N. Leklou, V. H. Nguyen, and P. Mounanga. The effect of the partial cement substitution with fly ash on Delayed Ettringite Formation in heat- cured mortars. KSCE Journal of Civil Engineering, 21(4):1359-1366, 2017.

[4] D. Damidot and F.P. Glasser. Thermodynamic investigation of the $\mathrm{CaO} \cdot \mathrm{Al}_{2} \mathrm{O}_{3} \cdot \mathrm{CaSO}_{4} \cdot \mathrm{H}_{2} \mathrm{O}$ system at $50^{\circ} \mathrm{C}$ and $85^{\circ} \mathrm{C}$. Cement and Concrete Research, 22(6):1179 - 1191, 1992.

[5] D. Damidot and F.P. Glasser. Thermodynamic investigation of the $\mathrm{CaO} \cdot \mathrm{Al}_{2} \mathrm{O}_{3} \cdot \mathrm{CaSO}_{4} \cdot \mathrm{H}_{2} \mathrm{O}$ system at $25^{\circ} \mathrm{C}$ and the influence of $\mathrm{Na}_{2} \mathrm{O}$. Cement and Concrete Research, 23(1):221 - 238, 1993.

[6] S. Diamond. Delayed ettringite formation - Processes and problems. Cement and Concrete Composites, 18(3):205215, 1996.

[7] P. K. Mehta. Mechanism of expansion associated with ettringite formation. Cement and Concrete Research, 3(1):1-6, 1973.

[8] G. Li, P. L. Bescop, and M. Moranville. Expansion mechanism associated with the secondary formation of the $U$ phase 
in cement-based systems containing high amounts of Na2SO4. Cement and Concrete Research, 26(2):195-201, 1996.

[9] H.F.W. Taylor. Sulfate reactions in concrete-microstructural and chemical aspects. Ceramic Transactions, 40 pp., 61, 1994.

[10] K Scrivener and J Skalny. International rilem workshop on internal sulfate attack and delayed ettringite formation. Materials and Structures, 37(1):71-72, 2004.

[11] X. Brunetaud. Étude de l'influence de différents paramètres et de leurs interactions sur la cinétique de l'amplitude de la réaction sulfatique interne au béton. $\mathrm{PhD}$ thesis, 2005. Thèse de doctorat dirigée par Damidot, Xavier Physicochimie des matériaux Châtenay-Malabry, Ecole centrale de Paris 2005.

[12] G. W. Scherer. Factors affecting crystallization pressure. In International RILEM Workshop on Internal Sulfate Attack and Delayed Ettringite Formation, pages 139154. RILEM Publications SARL, 2004.

[13] B. Budiansky and R. J. O'connell. Elastic moduli of a cracked solid. International Journal of Solids and Structures, 12(2):81 - 97, 1976.

[14] L. Dormieux, A. Molinari, and D. Kondo. Micromechanical approach to the behavior of poroelastic materials. Journal of the Mechanics and Physics of Solids, 50(10):2203-2231, 2002.
[15] L. Dormieux and D. Kondo. Exact solutions for an elastic damageable hollow sphere subjected to isotropic mechanical loadings. International Journal of Mechanical Sciences, 90:25-32, 2015.

[16] B. Pichler and L. Dormieux. Consistency of homogenization schemes in linear poroelasticity. C. R. Mecanique, 336:636-642, 2008.

[17] L. Dormieux and D. Kondo. Poroelasticity and damage theory for saturated cracked media, pages 153-186. Springer Vienna, Vienna, 2005.

[18] L. Dormieux, D. Kondo, and F.-J. Ulm. A micromechanical analysis of damage propagation in fluid-saturated cracked media. C. R. Mecanique, 334:440-446, 2006.

[19] Q.Z. Zhu, D. Kondo, and J.F. Shao. Micromechanical analysis of couppling between anisotropic damage and friction in quasi-brittle materials: Role of the homogenization scheme. International Journal of Solids and Structures, 45:13851405, 2008.

[20] V.-H. Nguyen, N. Leklou, J.-E. Aubert, and P. Mounanga. The effect of natural pozzolan on delayed ettringite formation of the heat-cured mortars. Construction and Building Materials, 48:479-484, nov 2013.

[21] R. A. Helmuth and D. H. Turk. Elastic moduli of hardened portland cement and tricalcium silicate pastes: effect of porosity. Technical report, 1966. 